\title{
Variations in/nearby genes coding for JAZF1, TSPAN8/LGR5 and HHEX-IDE and risk of type 2 diabetes in Han Chinese
}

\author{
Dai-zhan Zhou ${ }^{1,2,8}$, Yun Liu ${ }^{3,8}$, Di Zhang ${ }^{1,2,8}$, Si-min Liu ${ }^{4,5,6}$, Lan Yu²,7, Yi-feng Yang ${ }^{1,2}$, Teng Zhao ${ }^{1,2}$, \\ Zhuo Chen ${ }^{1,2}$, Meng-yuan Kan ${ }^{1,2}$, Zuo-feng Zhang ${ }^{4}$, Guo-yin Feng ${ }^{1,2}$, $\mathrm{He} \mathrm{Xu}^{1,2,4,5,6}$ and Lin $\mathrm{He}^{1,2,3}$
}

Several genetic loci (JAZF1, CDC123/CAMK1D, TSPAN8/LGR5, ADAMTS9, VEGFA and HHEX-IDE) were identified to be significantly related to the risk of type 2 diabetes and quantitative metabolic traits in European populations. Here, we aimed to evaluate the impacts of these novel loci on type 2 diabetes risk in a population-based case-control study of Han Chinese (1912 cases and 2041 controls). We genotyped 13 single-nucleotide polymorphisms (SNPs) in/near these genes and examined the differences in allele/genotype frequency between cases and controls. We found that both IDE rs 11187007 and HHEX rs1111875 were associated with type 2 diabetes risk (for both variants: odds ratio (OR) $=1.15,95 \%$ confidence interval $(\mathrm{Cl})$ 1.04-1.28, $P=0.009$ ). In a meta-analysis where we pooled our data with the three previous studies conducted in East Asians, we found that the variants of JAZF1 rs864745 (1.09 (1.03-1.16); $\left.P=3.49 \times 10^{-3}\right)$ and TSPAN8/LGR5 rs7961581 (1.11 (1.05-1.17); $P=1.89 \times 10^{-4}$ ) were significantly associated with type 2 diabetes risk. In addition, the meta-analysis (7207 cases and 8260 controls) also showed that HHEX rs1111875 did have effects on type 2 diabetes in Chinese population $\left(O R=1.15(1.10-1.21) ; P=1.93 \times 10^{-8}\right)$. This large population-based study and meta-analysis further confirmed the modest effects of the JAZF1, TSPAN8/LGR5 and HHEX-IDE loci on type 2 diabetes in Chinese and other East Asians. Journal of Human Genetics (2010) 55, 810-815; doi:10.1038/jhg.2010.117; published online 7 October 2010

Keywords: association study; Han Chinese population; HHEX; type 2 diabetes

\section{INTRODUCTION}

Type 2 diabetes is a prevalent chronic disease caused by the complex interplay of multiple genetic variants and environmental factors. In the past two decades, a total of 18 loci have been confirmed as type 2 diabetes risk loci with modest effect sizes in populations of European descent. ${ }^{1,2}$ Although most of these same loci have been confirmed to be associated with type 2 diabetes in Chinese, ${ }^{3-6}$ differences in allele frequency, genetic background, lifestyle and environmental exposures may lead to inconsistent results or different effect size of some genetic variants on type 2 diabetes risk between Chinese and European (for example, TCF7L2, HHEX-IDE, JAZF1, CDC123/CAMK1D, TSPAN8/LGR5). ${ }^{3,6}$

The association between the five loci (JAZF1, CDC123/CAMK1D, TSPAN8/LGR5, VEGFA and ADAMTS9) and type 2 diabetes were first identified by Zeggini et al. ${ }^{7}$ with meta-analysis of three genome-wide association studies. Subsequently, several replication studies indicated that most of the novel type 2 diabetes genes (JAZF1, TSPAN8/LGR5, $C D C 123 / C A M K 1 D$ and so on) appeared to affect various specific aspects of $\beta$-cell function. ${ }^{8-10}$ However, no significant association was detected for single-nucleotide polymorphisms (SNPs) from JAZF1, TSPAN8-LGR5, THADA, ADAMTS9, NOTCH2 in the previous Han Chinese study. ${ }^{3}$ Replication studies in other Asian populations just identified marginal significance of type 2 diabetes risk associated with some of these novel loci. ${ }^{11-13}$ We thought that the possible reasons for no or marginal significance were the limited sample size of these Asian population studies. To identify the effects of these genes on type 2 diabetes, we conducted a meta-analysis by pooling our data with those reported in the previous studies of East Asians.

HHEX-IDE locus was located at $10 \mathrm{q}$ region, which has previously been linked to fasting glucose, HbAlc levels and type 2 diabetes. ${ }^{14}$ Both HHEX and IDE genes have strong biological claims for a role in type 2 diabetes pathogenesis. However, all significant variants of this

\footnotetext{
${ }^{1}$ Institute for Nutritional Sciences, Shanghai Institutes for Biological Sciences, Chinese Academy of Sciences, Shanghai, PR China; ${ }^{2}$ Bio-X Center, Key Laboratory of Developmental Genetics and Neuropsychiatric Diseases (Ministry of Education), Shanghai Jiao Tong University, Shanghai, PR China; ${ }^{3}$ Institutes of Biomedical Sciences, Fudan University, Shanghai, PR China; ${ }^{4}$ Department of Epidemiology, UCLA, Los Angeles, CA, USA; ${ }^{5}$ Department of Medicine, UCLA, Los Angeles, CA, USA; ${ }^{6}$ Center for Metabolic Disease Prevention, UCLA, Los Angeles, CA, USA and ${ }^{7}$ Department of Psychiatry and Behavioral Sciences, School of Medicine, Johns Hopkins University, Baltimore, MD, USA 8These authors contributed equally to this work.

Correspondence: Dr H Xu or Dr L He, Institute for Nutritional Sciences, Shanghai Institutes for Biological Sciences, Chinese Academy of Sciences, Graduate School of the Chinese Academy of Sciences, 294 Taiyuan Road, Shanghai 200031, PR China.

E-mails: hexu@ucla.edu or helin@bio-x.cn
}

Received 27 June 2010; revised 4 August 2010; accepted 20 August 2010; published online 7 October 2010 
locus identified by genome-wide association studies of different ethnic groups were all located in $3^{\prime}$ region of HHEX gene, ${ }^{15-18}$ therefore, some studies suggested that HHEX was the most likely causal candidate gene for type 2 diabetes in chromosome 10q23-q25 ${ }^{19}$ and paid much attention to the HHEX gene. Here, eight common tag-SNPs, which covered both HHEX and IDE genes, were genotyped to analyze their effects on type 2 diabetes. Moreover, we also conducted a meta-analysis only in Chinese population to examine rs1111875 type 2 diabetes risk for the inconsistent results of previous studies.

\section{MATERIALS AND METHODS \\ Participants}

A total of 3953 individuals, Han Chinese, were enrolled in the current analysis from Shanghai, China. ${ }^{20}$ An interview was conducted by trained physicians or public health workers from the Pudong and Baoshan Centers for Disease Control and Prevention. The study individuals need to meet the following two criteria: (1) they were stable residents in the areas and (2) they were free from severe psychological disorders, physical disabilities and diagnosed with cancer, tuberculosis, AIDS and other communicable diseases within 6 months. Written informed consent was obtained from all the participants.

A total of 1912 type 2 diabetes patients ( 785 men, 1127 women; age $63.9 \pm 9.5$ years) were defined in accordance with World Health Organization criteria (fasting plasma glucose (FPG) $\geqslant 7.0 \mathrm{mmoll}^{-1}$ and/or 2-hour plasma glucose $\left.\geqslant 11.1 \mathrm{mmoll}^{-1}\right)$. Moreover, more than $50 \%$ of type 2 diabetes patients were diagnosed at the age of 45-65 years and have 5-15 years duration of disease. In all, 2041 controls ( 635 men, 1406 women; age $58.1 \pm 9.4$ years), ${ }^{4,20,21}$ with a FPG concentration $<6.1 \mathrm{mmoll}^{-1}$, were enrolled from the same geographical area with cases. The overall age and sex distribution of controls were matched with that of case group.

Height, weight, hip and waist circumference, and blood pressure were measured by trained medical professionals using a standardized protocol. Body mass index (BMI) was calculated as weight $(\mathrm{kg})$ per (height $(\mathrm{m}))^{2}$. Total cholesterol, high-density lipoprotein cholesterol, low-density lipoprotein cholesterol, triglycerides, HbAlc and FPG were measured enzymatically according to standard methods using Roche modular P800 autoanalyzer (Roche, Mannheim, Germany) with reagents (Roche Diagnostics $\mathrm{CmbH}$ ). Data are showed as medians $(25-75 \%$ range) or means \pm s.d. (Supplementary Table 1). High molecular weight genomic DNA was prepared from venous blood using the QuickGene 610L Automatic DNA/RNA Extraction System (Fujifilm, Tokyo, Japan).

\section{SNPs selection}

A total of 13 SNPs were genotyped, providing at least one representative SNP for each of the seven genes previously reported to be of some genome-wide significance in Europeans. These included five SNPs (rs864745 in JAZF1, rs7961581 in TSPAN8/LGR5, rs12779790 in CDC123/CAMK1D, rs4607103 in ADAMTS9 and rs9472138 in VEGFA) with minor allele frequency $>5 \%$ (7). For the HHEX-IDE locus, only representative SNPs with $r^{2}<0.80$ based on HapMap Han Chinese were selected for genotyping, and eight variants (rs1111875, rs2275729, rs5015480, rs7923837, rs1887922, rs2209772, rs1999763 and rs11187007) which had positive association with type 2 diabetes or diabetes-related traits in previous studies were selected. ${ }^{15-18,22-26}$

\section{Genotyping}

All 13 variants were genotyped using TaqMan genotyping assay on an ABI7900 system (Applied Biosystems, Foster City, CA, USA). Genotyping success rates were above $95 \%$ except rs12779790 and all mismatch rates were below $1 \%$ in duplicate samples.

\section{Statistical analysis}

We first evaluated the Hardy-Weinberg equilibrium for specific allele-genotype frequencies between cases and controls. We then calculated linkage disequilibrium (LD) coefficients $\left(\mathrm{D}^{\prime}\right.$ and $\left.r^{2}\right)$ in the control group. ${ }^{27}$ The association of all SNPs with type 2 diabetes was then examined by logistic regression adjusting for sex, age and loge-transformed BMI. We conducted a meta-analysis using comprehensive Meta Analysis software (Version 2.0, BIOSTAT, and Englewood, NJ, USA). Cochran's Q-test was performed to assess heterogeneity across different studies. For quantitative traits in relation to genotypes, a general linear statistical methodology was used, applying additive, dominant and recessive models while adjusting for the effect of age and sex (BMI, waist-tohip ratio), or age, sex and loge-transformed BMI (all other traits). The statistical analyses were performed by using the SPSS program (SPSS, Chicago, IL, USA). A $P$-value of $<0.05$ was considered significant.

\section{RESULTS}

The genotype distributions of each SNP selected for the present study (see Methods) in the case and control groups were shown in Table 1. All 13 SNPs genotyped were in Hardy-Weinberg equilibrium among controls (Table 1). We further compared frequencies of the reported risk allele in the Chinese populations with those in the Japanese, Indian and European populations (Table 2 and Supplementary Table 2). The allele frequencies for most of the SNPs examined in the present study were considerably different from the populations of Indian and European descent, but close to the Japanese population.

As shown in Table 1, rs7961581 in TSPAN8/LGR5 showed allelic difference between case and control groups (odds ratio $(\mathrm{OR})=1.13$, $95 \%$ confidence interval (CI) $1.01-1.26, P=0.031$ ). After adjusting with age, gender and loge-transformed BMI, rs864745 and rs7961581 tend to have the same direction of the association with type 2 diabetes ( $P=0.085$ and 0.055 , respectively), as previously reported in European genome-wide association studies. ${ }^{7}$ However, we could not observe any association between other SNP loci (rs12779790, rs4607103, rs9472138) and type 2 diabetes (nominal $P>0.05$ ). Similarly, subsequent meta-analyses (including six populations of $>10000$ individuals $)^{3,11,13}$ only revealed that rs864745 $(\mathrm{OR}=1.09,95 \% \mathrm{CI} 1.03-1.16$, $\left.P=3.49 \times 10^{-3}\right)$ and $\mathrm{rs} 7961581 \quad(\mathrm{OR}=1.11,95 \%$ CI $1.05-1.17$, $P=1.89 \times 10^{-4}$ ) were significantly associated with type 2 diabetes risk in East Asian populations (Table 3).

For the HHEX-IDE locus, the LD coefficients ( $\mathrm{D}^{\prime}$ and $r^{2}$ ) (Supplementary Table 3) suggested low pair-wise LD among most of the eight SNPs. However, rs11187007 in IDE and rs1111875 in HHEX, although which were about $250 \mathrm{~kb}$ apart from each other, were in a high LD $\left(\mathrm{D}^{\prime}=0.90, r^{2}=0.77\right)$. Meanwhile, both $\mathrm{rs} 11187007$ and $\mathrm{rs} 1111875$ were identified to be associated with type 2 diabetes risk after further adjustment for age, gender and BMI (for both variants: $\mathrm{OR}=1.15$, $95 \%$ CI $1.04-1.28, P=0.009$ in an additive model; Table 1). In addition, meta-analysis of six populations, total individuals of 15467 Chinese (7207 cases and 8260 controls) $)^{3,5,6,28}$ confirmed the association of the HHEX rs1111875 with type 2 diabetes $(\mathrm{OR}=1.15$, $95 \%$ CI $1.10-1.21, P=1.93 \times 10^{-8}$ ) (Table 3).

In analysis of quantitative traits, rs7923837, rs2275729, rs1111875 and rs11187007 appeared to affect plasma triglyceride levels in our Chinese participants, whereas rs7923837 showed effects on FPG. However, none of these associations still reach conventional statistical significance after adjusting for multiple comparisons (Supplementary Table 4).

\section{DISCUSSION}

In the present study, we identified a variant (rs11187007) in the $I D E$ gene with increased risk of type 2 diabetes and confirmed the effects of HHEX-IDE locus on type 2 diabetes in Chinese population. Our meta-analysis pooling data emanated from East Asians indicated that the JAZF1 and TSPAN8 loci may confer modest susceptibility to type 2 diabetes risk, as identified in the European decent populations. 
Table 1 Association of candidate SNPs with type 2 diabetes in case and control individuals

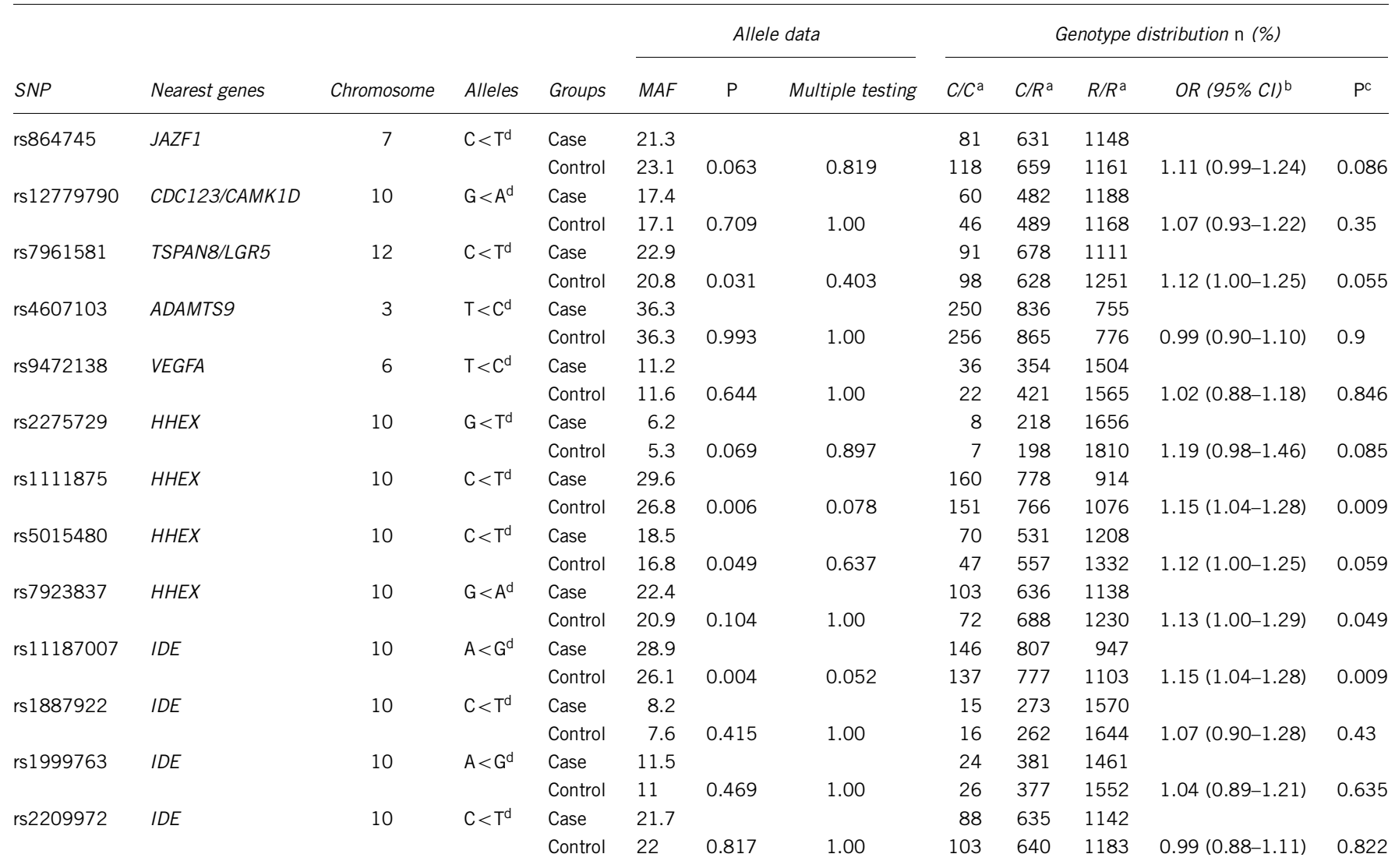

Abbreviations: $\mathrm{Cl}$, confidence interval; MAF, minor allele frequency; OR, odds ratio; SNP, single-nucleotide polymorphism.

${ }^{a} \mathrm{C} / \mathrm{C}$, homozygous for minor allele; C/R, heterozygous; R/R, homozygous for major allele.

bOR for original risk alleles reported in European populations.

cORs and $P$-values were adjusted for age, gender and loge-transformed BMI under the additive model.

${ }^{\mathrm{d}}$ Risk allele in the European populations.

Table 2 Comparison of risk-allele frequencies in the Chinese populations with those in other ethnic populations in previous studies

\begin{tabular}{|c|c|c|c|c|c|c|}
\hline SNPS & $\begin{array}{c}\text { Risk } \\
\text { allele }^{\mathrm{a}}\end{array}$ & Nearest genes & Chinese & $J_{a p a n e s e}{ }^{b}$ & Indians ${ }^{c}$ & Europeans ${ }^{\mathrm{d}}$ \\
\hline rs864745 & $\mathrm{T}$ & $J A Z F 1$ & 76.9 & 78.4 & 67.5 & 50.1 \\
\hline rs7961581 & C & TSPAN8/LGR5 & 20.8 & 20.5 & 35.2 & 26.9 \\
\hline rs12779790 & G & CDC123/CAMK1D & 17.1 & 14.7 & 12.6 & 18.3 \\
\hline rs4607103 & C & ADAMTS9 & 63.7 & 61 & 49.9 & 76.1 \\
\hline rs9472138 & $\mathrm{T}$ & VEGFA & 11.6 & 10 & NA & 28.2 \\
\hline
\end{tabular}

Abbreviations: NA, not available; SNP, single-nucleotide polymorphism.

aRisk allele in the European populations.

${ }^{6}$ Risk-allele frequencies previously reported by Omori et al. ${ }^{11}$

'Risk-allele frequencies previously reported by Sanghera et al. ${ }^{12}$

${ }^{\mathrm{d}}$ Risk-allele frequencies across the DGI/FUSION/UK stage 2 studies, previously reported by Zeggini et al. ${ }^{7}$

Although Florez et al. ${ }^{29}$ previously conducted a well-designed large-scale study and reported that genetic variants in the human IDE gene did not contribute to susceptibility of type 2 diabetes, available evidence does support that IDE has strong functional roles in type 2 diabetes' pathogenesis. The IDE gene encodes a metallopeptidase, which can degrade insulin, initiate cellular insulin processing and terminate its action. Homozygous mice with IDE gene deletion resulted in hyperinsulinemia and glucose intolerance, which are classical hallmarks in type 2 diabetes develop- ment. ${ }^{30}$ Here, we found a novel association of rs11187007 with type 2 diabetes and triglycerides, in line with the findings that variants and haplotypes in IDE were associated with FPG, HbAlc and type 2 diabetes. $^{22,23,30,31}$

On the other hand, the association study and meta-analysis further confirmed the effect of HHEX rs1111875 on type 2 diabetes in Chinese population, consistent with previous genome-wide association studies and replication studies in other ethnic populations. ${ }^{15-19,25,26,32-34}$ The HHEX gene encodes a transcription factor that is also involved in the Wnt signaling pathway and that has been shown to be essential for pancreas development. van Vliet-Ostaptchouk et al. ${ }^{19}$ suggested that HHEX was the most likely causal candidate in chromosome 10q. However, in our study, both IDE rs11187007 and HHEX rs1111875 were detected to be associated with type 2 diabetes. It also shows that a $270-\mathrm{kb}$ LD block covers the whole HHEX-IDE locus in the HapMap date of Chinese subjects. Moreover, the SNPs do not reside within the coding or putative regulatory regions of any gene. Therefore, it is difficult to explain which gene may be the susceptibility gene. Given the consistency of the association between the HHEX-IDE locus and type 2 diabetes risk observed in multiple populations, it does seem warranted to conduct deep sequencing of this region to identify potential causal variants in these two genes that may directly affect type 2 diabetes risk.

The JAZF1 gene encodes a transcriptional repressor of NR2C2 (nuclear receptor subfamily 2, group $\mathrm{C}$, ember 2). Nr2c2-/- knock 
Table 3 Meta-analysis of the association of candidate SNP loci with type 2 diabetes

\begin{tabular}{|c|c|c|c|c|c|c|c|c|c|c|c|}
\hline \multirow[b]{2}{*}{ SNPS } & \multirow[b]{2}{*}{ Studies (ethnic) ${ }^{a}$} & \multicolumn{3}{|c|}{ Type 2 diabetes ${ }^{\mathrm{b}}$} & \multicolumn{3}{|c|}{ Normal controls ${ }^{\mathrm{b}}$} & \multirow[b]{2}{*}{$P(Q)^{c}$} & \multicolumn{3}{|c|}{ Meta-analysis } \\
\hline & & $\mathrm{C} / \mathrm{C}$ & $C / R$ & $R / R$ & $\mathrm{C} / \mathrm{C}$ & $C / R$ & $R / R$ & & $O R^{C}$ & $95 \% \mathrm{Cl}$ & $P^{d}$ \\
\hline rs864745 (T>C) & Replication 1 (Japanese) & 64 & 522 & 1009 & 52 & 320 & 612 & & 1.07 & $(0.94-1.23)$ & \\
\hline \multirow[t]{6}{*}{ JAZF1 chromosome 7} & Replication 2 (Japanese) & 49 & 411 & 793 & 42 & 262 & 532 & & 1.02 & $(0.88-1.19)$ & \\
\hline & Replication 3 (Japanese) & 19 & 136 & 310 & 49 & 321 & 557 & & 1.27 & (1.04-1.55) & \\
\hline & Replication 4 (Japanese) & NA & NA & NA & NA & NA & NA & & 1.08 & $(0.95-1.22)$ & \\
\hline & All Japanese & & & & & & & 0.394 & 1.09 & $(1.01-1.17)$ & 0.023 \\
\hline & Replication 6 (Chinese) & 81 & 631 & 1148 & 118 & 659 & 1161 & & 1.11 & (0.99-1.23) & \\
\hline & All (Japanese + Chinese) & & & & & & & 0.549 & 1.09 & $(1.03-1.16)$ & $3.49 \times 10^{-3}$ \\
\hline rs7961581 (T>C), & Replication 1 (Japanese) & 81 & 540 & 966 & 49 & 317 & 606 & & 1.05 & $(0.91-1.20)$ & \\
\hline \multirow[t]{7}{*}{ TSPAN8/LGR5 chromosome 12} & Replication 2 (Japanese) & 63 & 418 & 777 & 36 & 252 & 553 & & 1.16 & (0.99-1.35) & \\
\hline & Replication 3 (Japanese) & 23 & 167 & 273 & 40 & 306 & 581 & & 1.14 & $(0.94-1.37)$ & \\
\hline & Replication 4 (Japanese) & NA & NA & NA & NA & NA & NA & & 1.12 & $(0.99-1.27)$ & \\
\hline & All Japanese & & & & & & & 0.786 & 1.11 & $(1.03-1.19)$ & $5.54 \times 10^{-3}$ \\
\hline & Replication 5 (Chinese) & NA & NA & NA & NA & NA & NA & & 1.08 & $(0.97-1.21)$ & \\
\hline & Replication 6 (Chinese) & 91 & 678 & 1111 & 98 & 628 & 1251 & & 1.13 & $(1.01-1.26)$ & \\
\hline & All (Japanese + Chinese) & & & & & & & 0.933 & 1.11 & $(1.05-1.17)$ & $1.89 \times 10^{-4}$ \\
\hline rs12779790 (A>G), & Replication 1 (Japanese) & 48 & 457 & 1101 & 25 & 244 & 718 & & 1.19 & $(1.02-1.39)$ & \\
\hline \multirow[t]{6}{*}{ CDC123/CAMK1D chromosome 10} & Replication 2 (Japanese) & 30 & 343 & 855 & 15 & 202 & 566 & & 1.13 & $(0.95-1.35)$ & \\
\hline & Replication 3 (Japanese) & 8 & 122 & 348 & 25 & 216 & 683 & & 1.00 & $(0.80-1.25)$ & \\
\hline & Replication 4 (Japanese) & NA & NA & NA & NA & NA & NA & & 0.98 & $(0.85-1.13)$ & \\
\hline & All Japanese & & & & & & & 0.271 & 1.07 & (0.99-1.17) & 0.094 \\
\hline & Replication 6 (Chinese) & 60 & 482 & 1188 & 46 & 489 & 1168 & & 1.02 & $(0.90-1.16)$ & \\
\hline & All (Japanese + Chinese) & & & & & & & 0.367 & 1.06 & (0.99-1.13) & 0.109 \\
\hline rs4607103 (C > T) & Replication 1 (Japanese) & 238 & 739 & 612 & 151 & 492 & 346 & & 0.92 & $(0.82-1.04)$ & \\
\hline \multirow[t]{7}{*}{ ADAMTS9 chromosome 3} & Replication 2 (Japanese) & 165 & 622 & 469 & 116 & 402 & 317 & & 1.00 & $(0.88-1.13)$ & \\
\hline & Replication 3 (Japanese) & 73 & 236 & 169 & 159 & 406 & 368 & & 1.05 & $(0.90-1.23)$ & \\
\hline & Replication 4 (Japanese) & NA & NA & NA & NA & NA & NA & & 1.09 & $(0.99-1.21)$ & \\
\hline & All Japanese & & & & & & & 0.186 & 1.02 & $(0.96-1.08)$ & 0.618 \\
\hline & Replication 5 (Chinese) & NA & NA & NA & NA & NA & NA & & 1.03 & $(0.93-1.14)$ & \\
\hline & Replication 6 (Chinese) & 250 & 836 & 755 & 256 & 865 & 776 & & 1.00 & $(0.91-1.10)$ & \\
\hline & All (Japanese + Chinese) & & & & & & & 0.461 & 1.01 & $(0.97-1.06)$ & 0.522 \\
\hline rs9472138 (C > T) & Replication 1 (Japanese) & 29 & 325 & 1259 & 7 & 173 & 808 & & 1.28 & $(1.08-1.54)$ & \\
\hline \multirow[t]{5}{*}{ VEGFA chromosome 6} & Replication 2 (Japanese) & 22 & 234 & 1009 & 6 & 154 & 678 & & 1.12 & $(0.92-1.37)$ & \\
\hline & Replication 3 (Japanese) & 5 & 69 & 407 & 8 & 181 & 744 & & 0.76 & $(0.58-1.00)$ & \\
\hline & All Japanese & & & & & & & 0.007 & 1.05 & (0.79-1.39) & 0.743 \\
\hline & Replication 6 (Chinese) & 36 & 354 & 1504 & 22 & 421 & 1565 & & 0.97 & $(0.84-1.11)$ & \\
\hline & All (Japanese + Chinese) & & & & & & & 0.008 & 1.03 & $(0.85-1.25)$ & 0.767 \\
\hline rs1111875 (T>C), & Ng et al. (Hongkong) ${ }^{28}$ & NA & NA & NA & NA & NA & NA & & 1.09 & $(0.98-1.21)$ & \\
\hline \multirow[t]{6}{*}{ HHEX chromosome 10} & Wu et al. (Beijing) ${ }^{6}$ & NA & NA & NA & NA & NA & NA & & 1.00 & $(0.81-1.25)$ & \\
\hline & Wu et al. (Shanghai) ${ }^{6}$ & NA & NA & NA & NA & NA & NA & & 1.64 & $(1.25-2.15)$ & \\
\hline & Hu et al. (Shanghai) ${ }^{3}$ & NA & NA & NA & NA & NA & NA & & 1.20 & (1.09-1.33) & \\
\hline & Tan et al. (Singapore) ${ }^{5}$ & NA & NA & NA & NA & NA & NA & & 1.15 & $(1.03-1.29)$ & \\
\hline & Present (Shanghai) & 914 & 778 & 160 & 1076 & 766 & 151 & & 1.15 & (1.04-1.28) & \\
\hline & All (Chinese) & & & & & & & 0.080 & 1.15 & $(1.10-1.21)$ & $1.93 \times 10^{-8}$ \\
\hline
\end{tabular}

Abbreviations: $\mathrm{Cl}$, confidence interval; NA, not available; OR, odds ratio; SNP, single-nucleotide polymorphism.

aData of replication 1, 2 and 3 in Japanese come from the study by Omori et al. ${ }^{11}$ Data of replication 4 in Japanese come from the study by Takeuchi et al. ${ }^{13}$ Data of replication 5 in Chinese come from the study by Hu et al. ${ }^{3}$ Data of replication 6 in Chinese come from the present study.

${ }^{\mathrm{b}} \mathrm{C} / \mathrm{C}$, homozygous for minor allele; $\mathrm{C} / \mathrm{R}$, heterozygous; R/R, homozygous for major allele.

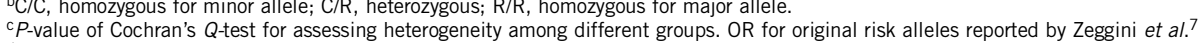

d $P$-value of the meta-analysis under the fixed model except rs9472138 (because of $P$-value of Cochran's $Q$-test $<0.05$ ).

out in mice retards growth and displays phenotypes of low serum concentrations of IGF1, hypoglycemia and reduced gluconeogenesis via PEPCK inactivation. ${ }^{7,35}$ Zeggini et al. ${ }^{7}$ have previously reported a strong association between rs864745 (in intron 1 of the JAZF1 gene) and type 2 diabetes risk in Europeans $\left(\mathrm{OR}=1.10, P=5.0 \times 10^{-14}\right)$. In the study by Grarup et al. ${ }^{9}$ it has been estimated that carriers of the T allele of JAZF1 rs864745 had a $2.6 \%(0.9-4.3 \% ; P=0.003)$ decreased insulin release, as assessed by the BIGTT acute insulin response index. In this article, the association studies and meta-analysis further confirmed the association between JAZF1 rs864745 and type 2 
diabetes in Chinese population. In addition, compared with the Europeans, the frequency of this risk variant in Chinese appeared to be much higher ( $\mathrm{T}$ allele, 0.77 vs 0.50 ).

We also observed significant association between TSPAN8/LGR rs7961581 and type 2 diabetes with a modest $\mathrm{OR}(\mathrm{OR}=1.11,95 \%$ CI 1.05-1.17) in East Asian populations, similar to that in European descents. In the study by Grarup et al., ${ }^{9}$ it was also reported that rs7961581 in TSPAN8 was associated with various OGTT-based surrogate measures of insulin release, suggesting a role of TSPAN8 in pancreatic $\beta$-cell function. In our meta-analysis, this variant also showed significant association with type 2 diabetes in Japanese population $\left(\mathrm{OR}=1.11,95 \%\right.$ CI $\left.1.03-1.19, P=5.54 \times 10^{-3}\right)$. Therefore, rs7961581, which resides $\sim 110 \mathrm{~kb}$ upstream of TSPAN8 (tetraspanin 8 ), is associated with type 2 diabetes across the boundary of race.

In the current study, polymorphisms (rs12779790, rs4607103 and rs9472138) showed any significant relations neither with type 2 diabetes risk nor with quantitative metabolic traits. Aside from the fact that genetic background may differ between Chinese and Europeans, differences in statistical power across studies may also explain such inconsistent observations, especially when the SNP-type 2 diabetes relation was modest. For example, we had a power of $\sim 99 \%$ to detect an OR of 1.15 for rs11187007 (given minor allele frequency $=0.30$ and $\sim 4000$ samples), but power declined to $79 \%$ for detecting an OR of 1.10.

In summary, in this large case-control study of type 2 diabetes among Han Chinese, the IDE rs11187007 and HHEX rs1111875 variants are significantly associated with increased type 2 diabetes risk. Our meta-analysis further confirmed the modest effects of the JAZF1, TSPAN8 and HHEX-IDE loci on type 2 diabetes risk in Chinese and other East Asians.

\section{ACKNOWLEDGEMENTS}

We thank the individuals who participated in the present study. This work was supported by grants from the National Key Technology R\&D Program (2006BAI05A05), the S973 Program (2006CB910601, 2007CB947300, 2010CB529600), 863 Program (2006AA02A407), the National Natural Science Foundation of China (30972529), the Opening Project of Shanghai Key Laboratory of Complex Prescription (07DZ22917), the Shanghai-Unilever Research and Development Fund (06SU07007), the Shanghai Leading Academic Discipline Project (B205), the Chinese Nutrition Society (05015), the Shanghai Municipality Science \& Technology Commission (05JC14090) and the Chinese Academy of Sciences (KSCX2-YW-R-01).

1 Prokopenko, I., McCarthy, M. I. \& Lindgren, C. M. Type 2 diabetes: new genes, new understanding. Trends Genet. 24, 613-621 (2008).

2 Stancakova, A., Kuulasmaa, T., Paananen, J., Jackson, A. U., Bonnycastle, L. L., Collins, F. S. et al. Association of 18 confirmed susceptibility loci for type 2 diabetes with indices of insulin release, proinsulin conversion, and insulin sensitivity in 5327 nondiabetic Finnish men. Diabetes 58, 2129-2136 (2009).

3 Hu, C., Zhang, R., Wang, C., Wang, J., Ma, X., Lu, J. et al. PPARG, KCNJ11, CDKAL1, CDKN2A-CDKN2B, IDE-KIF11-HHEX, IGF2BP2 and SLC30A8 are associated with type 2 diabetes in a Chinese population. PLOS ONE 4, e7643 (2009).

4 Liu, Y., Zhou, D. Z., Zhang, D., Chen, Z., Zhao, T., Zhang, Z. et al. Variants in KCNQ1 are associated with susceptibility to type 2 diabetes in the population of mainland China. Diabetologia 52, 1315-1321 (2009).

5 Tan, J. T., Ng, D. P., Nurbaya, S., Ye, S., Lim, X. L., Leong, H. et al. Polymorphisms identified through genome-wide association studies and their associations with type 2 diabetes in Chinese, Malays, and Asian-Indians in Singapore. J. Clin. Endocrinol. Metab. 95, 390-397 (2009).

6 Wu, Y., Li, H., Loos, R. J., Yu, Z., Ye, X., Chen, L. et al. Common variants in CDKAL1, $C D K N 2 A / B, I G F 2 B P 2, S L C 3 O A 8$, and HHEXIDE genes are associated with type 2 diabetes and impaired fasting glucose in a Chinese Han population. Diabetes 57, 2834-2842 (2008).
7 Zeggini, E., Scott, L. J., Saxena, R., Voight, B. F., Marchini, J. L., Hu, T. et al. Meta-analysis of genome-wide association data and large-scale replication identifies additional susceptibility loci for type 2 diabetes. Nat. Genet. 40, 638-645 (2008).

8 Boesgaard, T. W., Gjesing, A. P., Grarup, N., Rutanen, J., Jansson, P. A., Hribal, M. L. et al. Variant near ADAMTS9 known to associate with type 2 diabetes is related to insulin resistance in offspring of type 2 diabetes patients-EUGENE2 study. PLOS ONE 4, e7236 (2009).

9 Grarup, N., Andersen, G., Krarup, N. T., Albrechtsen, A., Schmitz, O., Jorgensen, T. et al. Association testing of novel type 2 diabetes risk alleles in the JAZF1, CDC123/ CAMK1D, TSPAN8, THADA, ADAMTS9, and NOTCH2 loci with insulin release, insulin sensitivity, and obesity in a population-based sample of 4516 glucose-tolerant middleaged Danes. Diabetes 57, 2534-2540 (2008).

10 Simonis-Bik, A. M., Nijpels, G., van Haeften, T. W., Houwing-Duistermaat, J. J., Boomsma, D. I., Reiling, E. et al. Gene variants in the novel type 2 diabetes loci CDC123/CAMK1D, THADA, ADAMTS9, BCL11A and MTNR1B affect different aspects of pancreatic beta cell function. Diabetes 59, 293-301 (2009).

11 Omori, S., Tanaka, Y., Horikoshi, M., Takahashi, A., Hara, K., Hirose, H. et al. Replication study for the association of new meta-analysis-derived risk loci with susceptibility to type 2 diabetes in 6244 Japanese individuals. Diabetologia. 52, 1554-1560 (2009).

12 Sanghera, D. K., Been, L., Ortega, L., Wander, G. S., Mehra, N. K., Aston, C. E. et al. Testing the association of novel meta-analysis-derived diabetes risk genes with type II diabetes and related metabolic traits in Asian Indian Sikhs. J. Hum. Genet. 54, 162-168 (2009).

13 Takeuchi, F., Serizawa, M., Yamamoto, K., Fujisawa, T., Nakashima, E., Ohnaka, K. et al. Confirmation of multiple risk loci and genetic impacts by a genome-wide association study of type 2 diabetes in the japanese population. Diabetes 58, 1690-1699 (2009).

14 Meigs, J. B., Panhuysen, C. I., Myers, R. H., Wilson, P. W. \& Cupples, L. A. A genomewide scan for loci linked to plasma levels of glucose and $\mathrm{HbA}(1 \mathrm{c})$ in a community-based sample of Caucasian pedigrees: the Framingham Offspring Study. Diabetes 51, 833-840 (2002).

15 Saxena, R., Voight, B. F., Lyssenko, V., Burtt, N. P., de Bakker, P. I., Chen, H. et al. Genome-wide association analysis identifies loci for type 2 diabetes and triglyceride levels. Science (New York, N.Y.) 316, 1331-1336 (2007).

16 Scott, L. J., Mohlke, K. L., Bonnycastle, L. L., Willer, C. J., Li, Y., Duren, W. L. et al. A genome-wide association study of type 2 diabetes in Finns detects multiple susceptibility variants. Science (New York, N.Y.) 316, 1341-1345 (2007).

17 Sladek, R., Rocheleau, G., Rung, J., Dina, C., Shen, L., Serre, D. et al. A genome-wide association study identifies novel risk loci for type 2 diabetes. Nature 445, 881-885 (2007).

18 WTCCC. Genome-wide association study of 14,000 cases of seven common diseases and 3000 shared controls. Nature 447, 661-678 (2007).

19 van Vliet-Ostaptchouk, J. V., Onland-Moret, N. C., van Haeften, T. W., Franke, L., Elbers, C. C., Shiri-Sverdlov, R. et al. HHEX gene polymorphisms are associated with type 2 diabetes in the Dutch Breda cohort. Eur. J. Hum. Genet. 16, 652-656 (2008).

20 Liu, Y., Yu, L., Zhang, D., Chen, Z., Zhou, D. Z., Zhao, T. et al. Positive association between variations in CDKAL1 and type 2 diabetes in Han Chinese individuals. Diabetologia 51, 2134-2137 (2008).

21 Zhou, D., Zhang, D., Liu, Y., Zhao, T., Chen, Z., Liu, Z. et al. The E23K variation in the KCNJ11 gene is associated with type 2 diabetes in Chinese and East Asian population. J. Hum. Genet. 54, 433-435 (2009).

22 Groves, C. J., Wiltshire, S., Smedley, D., Owen, K. R., Frayling, T. M., Walker, M. et al. Association and haplotype analysis of the insulin-degrading enzyme (IDE) gene, a strong positional and biological candidate for type 2 diabetes susceptibility. Diabetes 52, 1300-1305 (2003).

23 Gu, H. F., Efendic, S., Nordman, S., Ostenson, C. G., Brismar, K., Brookes, A. J. et al. Quantitative trait loci near the insulin-degrading enzyme (IDE) gene contribute to variation in plasma insulin levels. Diabetes 53, 2137-2142 (2004).

24 Mueller, J. C., Riemenschneider, M., Schoepfer-Wendels, A., Gohlke, H., Konta, L., Friedrich, P. et al. Weak independent association signals between IDE polymorphisms, Alzheimer's disease and cognitive measures. Neurobiol. Aging 28, 727-734 (2007).

25 Steinthorsdottir, V., Thorleifsson, G., Reynisdottir, I., Benediktsson, R., Jonsdottir, T., Walters, G. B. et al. A variant in CDKAL1 influences insulin response and risk of type 2 diabetes. Nat. Genet. 39, 770-775 (2007).

26 Zeggini, E., Weedon, M. N., Lindgren, C. M., Frayling, T. M., Elliott, K. S., Lango, H. et al. Replication of genome-wide association signals in UK samples reveals risk loci for type 2 diabetes. Science (New York, NY) 316, 1336-1341 (2007).

27 Shi, Y. Y. \& He, L. SHEsis, a powerful software platform for analyses of linkage disequilibrium, haplotype construction, and genetic association at polymorphism loci. Cell Res. 15, 97-98 (2005).

28 Ng, M. C., Park, K. S., Oh, B., Tam, C. H., Cho, Y. M., Shin, H. D. et al. Implication of genetic variants near TCF7L2, SLC30A8, HHEX, CDKAL1, CDKN2A/B, IGF2BP2, and FTO in type 2 diabetes and obesity in 6,719 Asians. Diabetes 57, 2226-2233 (2008).

29 Florez, J. C., Jablonski, K. A., McAteer, J., Sandhu, M. S., Wareham, N. J., Barroso, I. et al. Testing of diabetes-associated WFS1 polymorphisms in the diabetes prevention program. Diabetologia 51, 451-457 (2008).

30 Farris, W., Mansourian, S., Chang, Y., Lindsley, L., Eckman, E. A., Frosch, M. P. et al. Insulin-degrading enzyme regulates the levels of insulin, amyloid beta-protein, and the beta-amyloid precursor protein intracellular domain in vivo. Proc. Natl Acad. Sci. U.S.A. 100, 4162-4167 (2003). 
31 Karamohamed, S., Demissie, S., Volcjak, J., Liu, C., Heard-Costa, N., Liu, J. et al. Polymorphisms in the insulin-degrading enzyme gene are associated with type 2 diabetes in men from the NHLBI Framingham Heart Study. Diabetes 52, 15621567 (2003).

32 Cauchi, S., Proenca, C., Choquet, H., Gaget, S., De Graeve, F., Marre, M. et al. Analysis of novel risk loci for type 2 diabetes in a general French population: the D.E.S.I.R. study. J. Mol. Med. (Berlin, Germany) 86, 341-348 (2008).

33 Omori, S., Tanaka, Y., Takahashi, A., Hirose, H., Kashiwagi, A., Kaku, K. et al. Association of CDKAL1, IGF2BP2, CDKN2A/B, HHEX, SLC3OA8, and KCNJ11 with susceptibility to type 2 diabetes in a Japanese population. Diabetes $\mathbf{5 7}, \mathbf{7 9 1 - 7 9 5}$ (2008).

34 Pivovarova, O., Nikiforova, V. J., Pfeiffer, A. F. \& Rudovich, N. The influence of genetic variations in HHEX gene on insulin metabolism in the German MESYBEPO cohort. Diabetes Metab. Res. Rev. 25, 156-162 (2009).

35 Collins, L. L., Lee, Y. F., Heinlein, C. A., Liu, N. C., Chen, Y. T., Shyr, C. R. et al. Growth retardation and abnormal maternal behavior in mice lacking testicular orphan nuclear receptor 4. Proc. Natl Acad. Sci. U.S.A. 101, 15058-15063 (2004).

Supplementary Information accompanies the paper on Journal of Human Genetics website (http://www.nature.com/jhg) 